\title{
COLM TÓIBÍN: ENTRE ESPACIOS GEOGRÁFICOS Y DE LA MEMORIA EN THE HEATHER BLAZING
}

\author{
María Isabel Arriaga ${ }^{64}$
}

Resumen: En su estudio de la novela, Bakhtín considera a la heteroglosia como la principal característica de la prosa de ficción, dado que cada enunciación se produce en un momento histórico particular y en un entorno social específico. La novela, género literario dialógico por excelencia, se constituye por una pluralidad de voces y discursos sociales que representan distintas perspectivas del mundo representado en la obra literaria. En este marco, los conceptos de tiempo y espacio se fusionan en unidades de sentido inseparables que Bakhtín denomina "cronotopos", núcleos de representación esenciales de la obra. La novela The Heather Blazing (1992), del escritor y periodista irlandés Colm Tóibín, se estructura en torno a tres cronotopos: del encuentro, el umbral y la ruta, cuyo análisis permite explorar las ambigüedades que se producen en la Irlanda de 1990 durante su proceso de modernización y reorganización institucional. El propósito de este trabajo es analizar representaciones de Irlanda contemporánea y de reconstrucción identitaria en la novela de Tóibín, en torno a las categorías de tiempo-espacio e identidad, que se enmarca en el proyecto de investigación "Literatura irlandesa: estudios socio-críticos y traducción como diálogo intercultural", de la Facultad de Ciencias Humanas, UNLPam. Los resultados preliminares de esta investigación permitirían avizorar la coexistencia del pasado y el presente en la Irlanda de los años '90 y en la vida de los personajes de Tóibín, quienes -al igual que su nación- deben negociar valores en una sociedad fragmentada, aceptar el pasado y superar las pérdidas para re-inventarse hacia un futuro superador del pasado.

Palabras claves: lugar; memoria; identidad; representaciones; literatura irlandesa.

64 Profesora en Inglés y Licenciada en Lengua y Literatura Inglesa por la Universidad Nacional de La Pampa (Argentina). Profesora de Literatura y Cultura de los países de habla inglesa (UNLPam). Miembro fundador de la Asociación de Estudios Irlandeses del Sur (AEISur). Facultad de Ciencias Humanas de la UNLPam. 
Abstract: In his study of the novel, Bakhtin considers heteroglossia as the main characteristic of prose fiction, since each enunciation is produced in a particular historical moment and a specific social context. The novel, the dialogic literary genre par excellence, is constituted by a plurality of voices and social discourses that represent different perspectives of the world represented in the literary work. In this context, the concepts of time and space fuse in inseparable units of meaning that Bakhtin denominates "chronotopes", essential knots of representation within the text. The novel The Heather Blazing (1992), by Irish writer and journalist Colm Tóibin, is structured around three main chronotopes: that of the encounter, of the threshold and that of the road, whose analysis allows to explore the ambiguities that took place in 1990s Ireland during its process of modernisation and institutional reorganisation. The purpose of this paper is to analyse representations of contemporary Ireland and of identity reconstruction in Tóibín's novel, considering the categories of time-space and identity. This work develops within the research Project "Irish Literature: socio-critical studies and translation as an intercultural dialogue", that is carried out by the Faculty of Humanities of the National University of La Pampa. The preliminary results of this research will allow to notice the coexistence of past and present in 1990s Ireland as well as in the life of Tóibín's protagonists, who - like their nation - must negotiate values in a fragmented society, accept the past and overcome its losses in order to re-invent themselves towards a future in which the past be overcome.

Key words: place; memory; identity; representations; Irish literature.

"El concepto de lugar nunca es meramente geográfico, sino también ideológico" (HARTE, 2010, p. 337). La expresión "sentido del lugar" - sense of place - implica un acercamiento personal a un lugar mediante un doble proceso: por un lado, está la interpretación subjetiva, personal que una persona hace del entorno en cuestión; y, por el otro, su reacción emocional ante él. Ésto se debe al hecho de que, al igual que nuestra personalidad, los lugares son únicos para los individuos. En este sentido, Yi-Fu Tuan (1979, p. 411) asevera que los sentimientos que los seres humanos desarrollamos por el lugar al que consideramos el "hogar" (home) - esto es, el sitio de la memoria (the locus of memory) - son difíciles de explicar. Los lugares son dinámicos y cambiantes pero, al igual que los seres humanos, mantienen una identidad propia. En otras palabras, los 
Colm Tóibín: entre espacios geográficos y de la memoria en The Heather Blazing | 227

"lugares" son localizaciones cargadas de historia y memoria, como así también de un profundo significado emocional y simbólico para nosotros.

Dentro de la Geografía Humanística, que reflexiona sobre los fenómenos geográficos con el propósito de alcanzar una mayor y mejor comprensión del hombre y su condición, la noción de "paisaje" - landscape - aparece como uno de los conceptos más discutidos en las últimas décadas. Las nociones de tiempo y espacio, esenciales en toda experiencia humana, se enlazan estrechamente en la noción de "paisaje", la cual se encuentra profundamente asociada a la literatura irlandesa. Los paisajes son fenómenos complejos que reflejan un amalgamiento de personas y lugares que es esencial para la configuración de la identidad, tanto individual como local, dado que conllevan una importante carga cultural y afectiva. Debido a que los paisajes evolucionan con el tiempo, los mismos adquieren diferentes significados, los cuales pueden ser analizados principalmente desde enfoques históricos, arqueológicos, geográficos y sociológicos. Sin importar cuán variado sea un paisaje, su carácter ayuda a definir a quienes lo habitan y a distinguir el sentido de lugar que diferencia una región de otra.

Las representaciones geográficas adquieren relevancia en lo literario porque reflejan los procesos de crecimiento y maduración de una nación. Así, en consonancia con el desarrollo económico-social que Irlanda experimentó en los años ' 80 y '90, con el fenómeno conocido como "el Tigre Celta" - The Celtic Tiger -, las tradicionales representaciones literarias rurales de Irlanda perdieron predominio en las últimas décadas del siglo XX, siendo reemplazadas por paisajes citadinos que dan cuenta de las varias transformaciones socio-políticas, económicas y culturales que Irlanda atravesó en la década del '90s, principalmente, y con posterioridad.

Un nuevo escenario socio-político se ha ido consolidando en el territorio irlandés en las últimas tres décadas, caracterizado por el éxito económico de Irlanda, su incorporación al mundo globalizado, un bajo índice de desempleo ante la llegada de empresas internacionales que se asentaron en la "isla esmeralda", como así también una mayor libertad ganada por algunos grupos considerados, hasta hace poco tiempo, como minorías, tales como las mujeres y los homosexuales. Estos fenómenos socio-culturales y económicos también han permitido a Irlanda superar el extenso período diaspórico que la afectara desde finales del siglo XVII y hasta mediados del XX, lo cual dejó al país estigmatizado con uno de los índices de emigración más altos de la historia. 
Dentro de este contexto socio-político reciente, nuevos patrones de identidad individual y colectiva han emergido, junto con nuevas formas culturales que muchas veces colisionan con creencias y valores tradicionales. Como resultado de ello, crisis de identidad, ambivalencias y contradicciones - propias de una transición profunda entre un pasado fuertemente enraizado y un presente desafiante de las costumbres y valores patriarcales - han desafiado a los irlandeses en este contexto de cambio social.

Así, a pesar de tratarse de construcciones ficcionales, las novelas aparecen como el género literario más útil para retratar varios aspectos de la realidad social en la cual son producidas o contextualizadas. Ésto ha dado un nuevo giro a la novela irlandesa, que se ha vuelto esencialmente urbana, aunque el binomio rural/urbano continúa dominando muchas novelas irlandesas contemporáneas, especialmente como contraste entre el pasado y el presente. En este contexto, la novela irlandesa contemporánea ocupa un espacio cultural especial caracterizado por un alto sentido de continuidad y ruptura. Continuidad, dado que los escritores irlandeses contemporáneos - al igual que sus predecesores - no pueden separar la realidad social y política que los rodea de su arte, puesto que esa realidad los condiciona y se apropian de ella, lo cual los convierte en "su voz y profeta" (O'CONNELL, 2009, p. 3). Ruptura, porque si bien "la novela irlandesa ha sido un género en el cual explorar el carácter de la nación y la naturaleza de la identidad nacional, un vehículo obligado para el debate ideológico" (PRAGA TERENTE, 2005, p. 6), desde finales del siglo XX los escritores irlandeses se hayan comprometidos con mostrar al mundo a la nueva Irlanda; una Irlanda segura e inclusiva, caracterizada por el progreso fabril, la prosperidad material, una educación liberal y una iglesia auto-reformada" (KIBERD, 1995 , p. $581-582)^{65}$

Sin dudas, esto ha contribuido significativamente a otorgarle a la ficción irlandesa contemporánea un nuevo ingrediente: una alta dosis de subjetividad y de localismo. Sin importar si los escritores irlandeses escriben sus obras en Irlanda o en el extranjero, lo cierto es que es el "sentido del lugar" - no sólo del espacio irlandés físico sino principalmente del espiritual - como así también al individuo irlandés como protagonista de su propia realidad, lo que todos ellos describen a través de la literatura. Por todo ello, la novela irlandesa contemporánea requiere ser leída contextualmente, dado que temas históricos, políticos y

${ }^{65}$ Mi traducción. 
Colm Tóibín: entre espacios geográficos y de la memoria en The Heather Blazing | 229

socio-culturales fuertemente incrustados en la identidad nacional irlandesa se hacen presente en la literatura gaélica: "la novela irlandesa sólo puede abordarse y valorarse dentro de un debate ideológico y cultural de amplio espectro" (PRAGA TERENTE, 2005, p. 5).

Colm Tóibín es uno de esos escritores irlandeses contemporáneos con un profundo sentido del lugar, lo cual se refleja en el hecho de que su novela The Heather Blazing está contextualizada en Enniscorthy (su pueblo natal) y la costa de Cush, lugares emocionalmente ligados a su infancia. Eamon Redmond, el protagonista de The Heather Blazing, un juez de la Corte de Dublin, también es oriundo de Enniscorthy, en el Condado de Wexford, que se encuentra ubicado entre las montañas y el mar, en la principal ruta nacional de Dublin hacia el muelle Rosslare. Prácticamente toda la costa de Wexford está formada por una serie de largas playas fácilmente accesibles, todas separadas entre sí por cortas distancias. La posición central de Wexford otorga al protagonista de Tóibín la posibilidad de moverse fácilmente entre la costa y Dublín, donde ha residido durante toda su vida adulta.

Estos viajes a lo largo de las rutas del condado adquieren gran significación en esta obra, ya que representan la fragmentación temporal que el protagonista experimenta, lo cual lo lleva a sentirse atrapado por sus recuerdos, por su pasado, una y otra vez. Descripciones vívidas del paisaje aparecen en la novela de Tóibín, en un intento de compartir la experiencia del paisaje irlandés con los lectores y transmitirles sus sensaciones:

El calor almizclado del día se asentaría ahora en una tarde cálida en Cush, las polillas revoloteando contra las pantallas de luces, y el rayo del faro de Tuskar Rock... Regresó a su escritorio y pensó en ello: la corta orilla al pie del acantilado, la arcilla de marga roja, la curva lenta de la línea costera yendo al sur a Ballyconnigar, Ballyvaloo, Curracloe, Raven's Point y más allá de ellos hacia los pueblos deslavados y la ciudad de Wexford (TÓIBÍN, 1992, p. 9) ${ }^{66}$.

${ }^{66}$ Mi traducción: The musky heat of the day would settle now into a warm evening in Cush, the moths flitting against the lightshades, and the beam from the lighthouse of Tuskar Rock ... He went back to his desk and thought about it: the short strand at the botton of the cliff, the red marl clay, the slow curve of 
Sin embargo, la mayor parte de la vida del protagonista no transcurre en Enniscorthy. Éste es sólo el lugar de la infancia y la adolescencia para Eamon, un lugar que como adulto trata de evitar cuando regresa a la casa de la playa cada verano. A pesar de que Eamon busca constantemente alejarse de la ciudad en la que creció, cada vez que visita a su anciana tía Margaret, quien continúa viviendo en la casa familiar de los Redmond, Eamon puede sentir la seguridad que emerge del sentido de lugar que su ciudad natal siempre le ha inspirado, ya que es allí donde se localizan las memorias más ricas - aunque en muchos casos cargadas de soledad y tristeza - de Eamon niño. Así, de regreso a Enniscorthy, mientras "Eamon conducía hacia Templeshannon sentía que siempre había estado allí; la repentina claridad de su reconocimiento hacía del resto del mundo como un lugar extraño y no familiar" (TÓIBÍN, 1992, p. 59) ${ }^{67}$.

Pero es justamente en la costa, próxima a su casa de descanso estival donde, alejado de su rutina y presiones laborales, Eamon se permite conectar con sí mismo, con sus afectos y sus recuerdos. Dado que el concepto de "lugar" implica asociaciones cercanas con los seres humanos, los lugares con los que estamos familiarizados pueden tener un rol enriquecedor en nuestras vidas porque aprendemos patrones de comportamiento y actitudes en ellos, interactuamos con otros y le otorgamos significado a esos lugares. Según Tuan, los individuos frecuentemente se vuelven conscientes de su apego (attachment) a un lugar una vez que han partido del mismo y pueden verlo desde la distancia, ya que este sentimiento se vuelve más agudo cuando sentimos añoranza (TUAN, 1979, p. 411). La costa erosionada contribuye a producir una fragmentación temporal en la secuencia narrativa de la novela, lo cual fuerza al protagonista a hacer un alto en su vida, y al lector, a unificar el texto en un nuevo todo.

El apego y la atracción que Eamon siente por la costa de Cush se ha desarrollado desde su temprana infancia, en los tiempos en que su padre lo llevaba a pasar cada verano a la playa, donde compartían buenos

the coastline going south to Ballyconnigar, Ballyvaloo, Curracloe, Raven's Point and beyond them to the sloblands and Wexford town.

${ }^{67}$ Mi traducción: "But as he drove into Templeshannon he felt that he had always been there; the sudden clarity of his recognition made the rest of the world strange and unfamiliar". 
momentos con algunos amigos de la familia. Es en la inmensidad y el aislamiento que el dramático paisaje costero ofrece donde Eamon recuerda a su ya fallecido padre: "el nadar ese día en el pasado, sus brazos alrededor del cuello de su padre, ${ }^{68}$ la textura de la piel húmeda de su padre y del agua estaban todavía con él” (TÓIBÍN, 1992, p. 55). Y es únicamente allí donde el protagonista de Tóibín también puede imaginar cómo era su madre, a quien nunca conoció. La escena del protagonista solitario en la costa irlandesa es una imagen recurrente en esta novela de Tóibín, ya que no sólo resalta la fuerte presencia del pasado en la mente del personaje principal en esos momentos sino que, además, determina verdaderos momentos de epifanía para él. Según Harte, "la condición de foraneidad solitaria en la ficción de Tóibín está estrechamente relacionada con sentimientos de pérdida que típicamente emergen de algún trauma infantil no hablado, cuya represión causa una resonancia psíquica duradera. Estas heridas psíquicas están asociadas a experiencias tempranas de muerte o abandono no resueltas, cuyos efectos diferidos demuestran ser profundamente determinantes de comportamientos susequentes" $^{69}$ (HARTE, 2010, p.334), tal como se muestra en The Heather Blazing.

Así, los lugares pueden ser significativos para los seres humanos porque proveen un sentido de continuidad a nuestras vidas o porque sirven como punto de partida para un cambio, y hasta pueden despertar en los seres humanos sentimientos de seguridad, pertenencia, autoexpresión o libertad. Esos sentimientos juegan un rol esencial en la formación y mantenimiento de las conexiones que los individuos establecen entre lugar e identidad, cuya naturaleza no es simple y pareciera crearse a partir de la experiencia repetida: "el patrón funcional de nuestras vidas es capaz de establecer un sentido del lugar. Al llevar a cabo nuestras rutinas diarias vamos regularmente de un punto a otro, siguiendo caminos establecidos, de modo que con el tiempo se forma una red de nudos cuya relación está impresa en nuestro sistema perceptual y afecta nuestras expectativas corporales" (TUAN, 1979, p. 418) ${ }^{70}$.

De esta manera, Tóibín transforma el espacio de la costa erosionada de Enniscorthy en un espacio de encuentro para el

\footnotetext{
${ }^{68} \mathrm{Mi}$ traducción: "the swim that day in the past, his arms around his father's neck, the texture of his father's wet skin and the thrill of the water were still with him" (TÓIBÍN, 1992, p. 55).

${ }^{69} \mathrm{Mi}$ traducción.

${ }^{70}$ Mi traducción.
} 


\section{2 | María Isabel Arriaga}

protagonista con ese pasado que por momentos lo atormenta, en un lugar de entendimiento, reconciliación y de toma de decisiones para seguir adelante. Tóibín siempre se ha sentido especialmente atraído por su paisaje local y ha capturado la importancia del lugar en mayor grado que muchos otros escritores de su propia nacionalidad y de otras. Con su combinación de aislamiento y belleza natural, el paisaje irlandés tiene un poder de encantamiento único, que ha servido como fuente de inspiración eterna para los escritores irlandeses quienes, aún escribiendo desde el exilio o en un lugar extranjero, siempre sitúan al menos parte de sus historias en Irlanda, principalmente en sus ciudades natales o en lugares que ellos perciben como "el hogar", como si sintieran una imperiosa necesidad de (re)conectar constantemente con sus raíces a través de la literatura.

En su estudio de la novela, Mikhail Bakhtin argumenta que la crítica de la novela necesita de referencias al contexto social del discurso, para que las distintas voces sociales e históricas que pueblan el lenguaje puedan emerger, otorgando diferentes perspectivas y conceptualizaciones del mundo (BAKHTIN, 1981, p. 678) ${ }^{71}$. La novela, a la que Bakhtin considera como el género dialógico por excelencia, presenta una fragmentación de perspectivas, debido a su naturaleza dialógica, a su "heteroglosia". Todos los lenguajes de lo que Bakhtin denomina heteroglosia (heteroglossia) representan "perspectivas específicas del mundo, formas de conceptualizar el mundo en palabras, cada una caracterizada por sus propios objetos, significados y valores" (p. 291$292)^{72}$. Según el lingüista ruso, la heteroglosia es la característica más importante de la prosa de ficción, debido a que cualquier enunciación se lleva a cabo en un momento histórico particular, en un contexto social específico. Por lo tanto, una vez incorporada a la novela, la heteroglosia se convierte en "el discurso de otro en el lenguaje de otro" (p.324). Ésto se debe a que Bakhtin entiende al lenguaje como "ideológicamente

${ }^{71}$ From "Discourse in the Novel" (1981): "The novel must be forced to reveal the social and historical voices populatin language ... which provide language with its particular concrete conceptualisations" (BAKHTIN, p. 678).

72 From "Discourse in the Novel" (1981): "Thus at any given moment of its historical existence, language is heteroglot from top to bottom: it represents the co-existence of socio-ideological contradictions between the present and the past, between differing epochs of the past, between different socio-ideological groups in the present, between tendencies, schools, circles and so forth, all given a bodily form. These 'languages' of heteroglossia intersect each other in a variety of ways, forming new socially typifying 'languages'" (BAKHTIN, p. 291). 
Colm Tóibín: entre espacios geográficos y de la memoria en The Heather Blazing | 233 saturado" (p. 271) y a este "dialogismo" como propiedad de cualquier discurso viviente (p. 279) y del mundo social. En este contexto, la literatura emerge no sólo como una forma discursiva en general sino, específicamente, como una práctica social, en la cual cada palabra enunciada conlleva una carga histórica, cultural y social.

Desde una perspectiva humanista, "el estudio del espacio es el estudio de los sentimientos espaciales de las personas en el continuo de la experiencia" (TUAN, 1979, p. 338), debido a que los seres humanos atribuimos significado a ciertos lugares en el proceso de construcción de nuestra identidad. El análisis del contexto socio-cultural de la novela requiere de dos elementos esenciales de la experiencia humana: tiempo y espacio, a los que Bakhtin no concibe como entes separados sino uno a partir de la existencia del otro ${ }^{10}$. En efecto, cada uno de estos conceptos se transforma en una dimensión del otro, cuya inseparabilidad se concretiza en su noción del "cronotopo" literario, al que Bakhtin define como "los nudos de la narración donde el tiempo se vuelve palpable y visible, donde el tiempo se materializa en el espacio, y emerge como el centro de concretización de la representación, que da cuerpo a toda la novela"73 (BAKHTIN, 1981, p. 250). En otras palabras, los cronotopos son para Bakhtin la columna vertebral de una narración, ya que la forma en que el tiempo y el espacio son representados por el cronotopo le permite al autor organizar la estructura de la narración y otorgarle sentido:

en el cronotopo artístico literario, los indicadores espaciales y temporales se fusionan en un todo cuidadosamente pensado, concreto. El tiempo, por así decirlo, se espesa, se encarna, se vuelve visible; del mismo modo, el espacio se carga y responde a los movimientos del tiempo, las tramas y la historia. La intersección de ejes y la fusión de indicadores

${ }^{73}$ The chronotope is where the knots of a narrative are tied and untied . . Time becomes, in effect, palpable and visible; the chronotope makes narrative events concrete, functioning as the primary means for materializing time in space, emerges as a center for concretizing representation, as a force giving body to the entire novel. All the novel's abstract elements - philosophical and social generalizations, ideas, analysis of cause and effect - gravitate towards the chronotope and through it take on flesh and blood, permitting the imagining power of art to do its work (BAKHTIN, 1981, p. 250). 
caracteriza al cronotopo artístico (BAKHTIN, 1981, p. $84)^{74}$.

De esta manera, aplicado al análisis literario, los cronotopos actúan como los hilos estructurales que subyacen en la composición de una novela, con una función representativa por excelencia. En la teoría Bakhtiniana, el cronotopo es la unidad elemental de la imaginación literaria y, por lo tanto, la piedra angular de la experiencia estética. Los cronotopos actúan como centros organizadores de los eventos principales en una novela, dado que definen el contexto espacial y temporal en el que la narración se lleva a cabo, y desempeñan un rol esencial en la construcción del significado. Además, los cronotopos no sólo definen la estructura interna de una novela sino que también contribuyen a conectar el texto literario con el contexto socio-cultural de una narración debido a la inseparabilidad de sus componentes en la creación artística.

Un cronotopo particular usualmente invoca otros tiempos y realidades. Dado que los cronotopos son motivos recurrentes en el texto, tres de los cronotopos principales analizados por Bakhtin en su estudio de la novela pueden aplicarse a la obra de Tóibín: el cronotopo de la ruta (road), el del encuentro (encounter) y el del umbral (threshold). Una imagen frecuente en The Heather Blazing es el cronotopo de la ruta, debido a los viajes que el protagonista y su esposa Carmel emprenden hacia su casa de la costa cada año al iniciarse el verano, en coincidencia con el receso de la Corte de Dublín. Aquí, tiempo y espacio se fusionan ya que el contacto con un paisaje familiar lleva la mente del protagonista al pasado. En la novela de Tóibín la imagen de la ruta mantiene similitudes con el rol que Bakhtin le asignara en su estudio: se trata de una ruta que atraviesa un territorio familiar para el protagonista, haciendo "el camino a casa" más significativo para el mismo, lo cual facilita la comunicación y la revelación de la verdad.

Otro cronotopo destacado en The Heather Blazing es el del encuentro. Bakhtin atribuye a este cronotopo un alto grado de intensidad emocional y evaluativa (BAKHTIN, 1981, p. 243), en el cual predomina el elemento temporal. Esta imagen cronotópica está relacionada con uno

${ }^{74}$ In the literary artistic chronotope, spatial and temporal indicators are fused into one carefully thought-out, concrete whole. Time, as it were, thickens, takes on flesh, becomes visible; likewise, space becomes charged and responsive to the movements of time, plots and history. This intersection of axes and fusion of indicators characterizes the artistic chronotope (BAKHTIN, 1981, p. 84). 
Colm Tóibín: entre espacios geográficos y de la memoria en The Heather Blazing | 235

de los motivos más universales de la literatura: el del encuentro interpersonal, que puede servir como inicio, culminación o desenlace del argumento de la historia. En esta novela, los encuentros se dan como desenlace (denouement) de la misma.

Por último, el cronotopo más relevante en The Heather Blazing es sin dudas el del umbral, "altamente cargado de emoción y valor" (BAKHTIN, 1981, p. 248), debido a que su protagonista enfrenta una identidad en crisis mientras atraviesa momentos de incertidumbre y ambivalencia por eventos no resueltos de su pasado, que han hecho de él un hombre solitario, poco comunicativo y con gran dificultad para aceptar los cambios y desafíos que la vida le plantea. El término "umbral" tiene un significado metafórico, relacionado con los momentos de crisis, con decisiones que cambian una vida, pero también con la indecisión que entorpece el proceso de cambio en los personajes por el temor que experimentan para cruzar ese umbral, para dar ese paso que marcará un antes y un después en la vida del protagonista. Un umbral es, por lo tanto, un espacio de transición, caracterizado por la incertidumbre y que implícitamente conlleva el potencial de un cambio radical. Eamon Redmond, de mediana edad, se encuentra en el umbral de su vida, enfrentando un estadío de madurez que inevitablemente lo fuerza a "cruzar el puente" y encontrar puntos de transición que lo llevarán a nuevas formas de identidad y a un acercamiento a su familia, con quienes siempre se ha mostrado distante por su incapacidad para mostrar sus sentimientos y afectos.

En la novela de Tóibín, el cronotopo del umbral está representado por dos imágenes recurrentes que atraviesan la obra y que son determinantes del estado psicológico y emocional del protagonista: por un lado, la imagen de la costa erosionada; por otro lado, la imagen de la muerte. En ambas, la presencia del mar vuelve al protagonista consciente de la idea de pérdida y del implacable poder de la muerte. El cronotopo marino aparece acompañado de la figura del observador aislado, que según Harte, actúa como una "consciencia gobernante en las ficciones de Tóibín”75 (HARTE, 2010, p. 334). Caminando solo por la playa, Eamon observa y evalúa los efectos que la erosión va causando en la costa año tras año, imagen que lo sumerge en el mundo de sus recuerdos, y en el pasado histórico de Irlanda. Después del fallecimiento de su esposa, esta imagen del observador solitario se intensifica, ya que el protagonista se encuentra

${ }^{75}$ Mi traducción. 
sumido en una profunda soledad y es únicamente mediante una intensa rutina de caminatas y natación que él mismo se fija como Eamon encuentra las fuerzas para continuar viviendo sin Carmel.

Según Harte, el apego que Eamon siente por el mar se relaciona con el hecho de que "la costa de Cush es el lugar donde fue llevado desde bebé después de la muerte de su progenitora, hecho que pareciera haber causado una suerte de transferencia emocional dentro de él, por la cual el mar se ha convertido para Eamon en un refugio maternal, en contraste con las casas, que son consistentemente representadas en esta novela como figuras desnudas, vacías, emblemas poco acogedores de la ausencia materna"76 (HARTE, 2010, p. 343). Así, cuanto más demandante se vuelve su rol en el sistema judicial irlandés, Eamon más necesita del contacto liberador que el agua le proporciona. Por otra parte, ante una nueva crisis determinada por una inminente muerte en la familia, Eamon siente que su vida se desmorona y es aquí donde nuevamente se refuerza la imagen cronotópica del umbral, que traerá aceptación ante nuevas pérdidas en la vida del protagonista.

Eamon Redmond se traslada a la costa cada verano para escapar de las presiones de su vida profesional en la Irlanda de los '90s, una Irlanda que está cambiando rápidamente, que se va modernizando a pasos agigantados y reorganizando a nivel político-institucional, todo lo cual lo obliga a cuestionarse los fundamentos de su identidad nacional, sus valores personales y sus convicciones profesionales.

The Heather Blazing muestra que el proceso de modernización que Irlanda atravesó en los años '90 - luego de casi un siglo de un sistema patriarcal fuertemente instalado desde el poder político hegemónico - no fue uniforme. Por el contrario, estuvo marcado por tensiones y contradicciones propias de una nación en (re)construcción. Así, mientras la obra refleja ciertas alternativas y transformaciones a los conceptos tradicionales de de familia e historia, como así también una mayor secularización de los poderes del estado, se observa que el poder judicial irlandés no se había modernizado en consonancia. En consecuencia, Eamon experimenta una profunda crisis profesional porque mientras redacta sus veredictos judiciales es consciente de que ya no tiene valores morales fuertes, que ha dejado de creer en todo (TÓIBÍN, 1992, p. 90) y, por ello, no se siente capacitado para resolver casos jurídicos que demandan mucho más que la fría aplicación de leyes obsoletas enmarcadas en la Constitución de 1937, leyes que ya no responden a las

\footnotetext{
${ }^{76}$ Mi traducción.
} 
necesidades contemporáneas de una sociedad en transformación. La pérdida de su fe, no sólo en Dios sino también en los mitos del nacionalismo irlandés - en cuyos fundamentos había sido educado -, hacen que su mundo colapse y entre en una crisis de identidad que sólo podrá ir resolviendo hacia el final de la novela, ante la presencia sanadora del mar y a través de su nueva relación con su pequeño nieto.

El contacto con un lugar que conoce muy bien desde su infancia lo llevan a ver su vida entera en retrospección, dado que el desplazamiento espacial que el protagonista experimenta cada verano produce en él una dislocación temporal. Tiempo y espacio confluyen en este cronotopo de manera indisoluble: la conciencia del paso del tiempo es materializada en el deterioro de la costa marina, que va siendo ganada por el avance implacable del mar, cuyo poder destructivo obliga al protagoista a enfrentar los desafíos y a aceptar lo inevitable. En un paisaje tan cambiante como es el irlandés, el mar aparece como la única fuerza permanente en la historia de Irlanda, en contraste con la tierra, y las nociones tradicionales de familia e historia, que se van marchitando en medio de las profundas transformaciones que la nación enfrenta en su apogeo de los años '90. Estos cambios que el protagonista observa - tanto en el paisaje natural como en el urbano - son utilizadas por Tóibín como metáforas creativas que reflejan cambios culturales significativos en la representación de lo que hasta ese momento era considerado como "lo irlandés" o Irishness. De esta manera, a través de la crisis personal de su protagonista, el autor muestra una Irlanda nueva, global, pujante y tolerante, en la que los pilares tradicionales del estricto nacionalismo irlandés - familia, iglesia y estado - empiezan a colapsar y a re-inventarse, del mismo modo que Irlanda lo ha empezado a hacer.

En conclusión, la naturaleza simbólica del cronotopo del umbral permite a Tóibín representar la situación particular que Irlanda atravesó en la última década del siglo XX, como una transición en la que valores del pasado y del presente conviven y hasta a veces se enfrentan, del mismo modo en que el pasado y el presente coexisten en la identidad fragmentada de su protagonista, y pujan por alcanzar un equilibrio reparador de las heridas del ayer. Desde este punto de vista, The Heather Blazing constituye una exploración crítica aunque moderada de las tensiones y ansiedades que emergen "en un estado más preocupado con ser que con convertirse" (HARTE, 2002, p. 55-56), en el cual los valores de una nación imaginada continúan luchando por sobrevivir por encima de otros que derivan de un complejo cambio social que parece haber alcanzado un punto sin retorno. Este estado de cosas responde también a 
las características de nuestros tiempos, donde según Stuart Hall "las identidades modernas se están de-centrando, dislocando, fragmentando" (HALL, 1996a, p. 597). En este contexto, no es extraño hablar de una crisis de identidad en la posmodenidad porque la identidad humana sólo puede ser entendida como "una construcción, como un proceso nunca completo” (HALL, 1996b, p. 2).

\section{BIBLIOGRAFÍA}

BAKHTIN, Mikhail. Michael HOLQUIST (Ed./ trans.) and Caryl Emerson (trans.). The Dialogical Imagination: Four Essays. Discourse in the Novel, and Forms of Time and of the Chronotope in the Novel. English version. Austin: University of Texas Press, 1981.

HALL, Stuart and Paul DU GAY (Ed). Questions of Cultural Identity. London, Thousand Oaks, New Delhi: Sage Publications Ltd, 1996b. HALL, Stuart, David HELD, Don HUBERT, and Kenneth THOMPSON (Eds). Modernity: an Introduction to Modern Societies. Great Britain: Blackwell publishers, 1996a.

HARTE, Liam. The Endless Mutation of the Shore Colm Tóibin's Marine Imagery. Critique. Vol. 51. № 4 (2010).

. History, Text, and Society in Colm Tóibín's The Heather Blazing. NewHibernia Review 6.4harte.pdf Project Muse ®. Volume 6. № 4 (2002).

KIBERD, Declan. Inventing Ireland: the Literature of the Modern Nation. Great Britain: Vintage, 1995.

O’CONNELL, Viviana. La Isla de la Poesía. Algunas Consideraciones sobre Literatura Irlandesa. Web. 15 Jan 2009. <http://www.almargen.com.ar/sitio/seccion/literatura/litirland/>

PRAGA TERENTE, Inés. La Novela Irlandesa del Siglo XX. Barcelona: PPU S.A., 2005.

TÓIBÍN, Colm. The Heather Blazing. London: Picador, 1992.

TUAN, Yi-Fu. Space and place: Humanistic Perspective (Introduction) in S. Gale, and G. Olsson (Eds), Philosophy of Geography, Dordrecht: Reidrel Publishing, 1979.

Recebido em: 15/02/2018

Aceito em: 29/03/2018 